\title{
Felice Dassetto (Ed.), Discours musulmans contemporains. Diversité et cadrages
}

Louvain-la-Neuve, Éditions Académia, 2011, 146 p.

\section{Cédric Baylocq}

\section{(2) OpenEdition}

\section{Journals}

Édition électronique

URL : http://journals.openedition.org/assr/25449

DOI : $10.4000 /$ assr. 25449

ISSN : $1777-5825$

Éditeur

Éditions de l'EHESS

Édition imprimée

Date de publication : 30 décembre 2013

Pagination : 172

ISSN : 0335-5985

Référence électronique

Cédric Baylocq, «Felice Dassetto (Ed.), Discours musulmans contemporains. Diversité et cadrages », Archives de sciences sociales des religions [En ligne], 164 | 2013, mis en ligne le 21 février 2014, consulté le 21 septembre 2020. URL : http://journals.openedition.org/assr/25449 ; DOI : https://doi.org/ 10.4000/assr.25449

Ce document a été généré automatiquement le 21 septembre 2020.

(c) Archives de sciences sociales des religions 


\section{Felice Dassetto (Ed.), Discours musulmans contemporains. Diversité et cadrages}

Louvain-la-Neuve, Éditions Académia, 2011, 146 p.

\section{Cédric Baylocq}

\section{RÉFÉRENCE}

Felice Dassetto (Ed.), Discours musulmans contemporains. Diversité et cadrages, Louvain-la-Neuve, Éditions Académia, 2011, 146 p. 
1 Ce petit ouvrage collectif de 140 pages (+ notules biographiques des auteurs et table des matières) se propose d'examiner un certain nombre de discours d'acteurs musulmans contemporains, exerçant principalement leur magistère en Europe. $\mathrm{Ce}$ recueil s'insère dans un axe de recherche plus large porté par le très dynamique Centre Interdisciplinaire d'étude de l'Islam dans le Monde Contemporain (CISMOC), dirigé par la sociologue Brigitte Maréchal, spécialiste $\mathrm{du}$ fait religieux musulman en Europe (particulièrement de la mouvance protéiforme Frères Musulmans). Cet axe de recherche s'intéresse à la production des discours des nouveaux acteurs du champ du leadership musulman en Europe.

2 La notion de « discours " semble être ici entendue au sens large des prises de positions des acteurs de ce champ sur des sujets relevant de la théologie, de la politique et du social, mais aussi des constructions théologiques et/ou canoniques un peu plus élaborées de certains d'entre eux (ici Tariq Ramadan, Abdelmajid Charfi, Asmae Lmrabet, ou encore Tahar Mahdi). Ces constructions nouvelles ont suscité de nombreux ouvrages d'analyse (plus denses et systématiques que celui commenté ici) ces dix dernières années, au rang desquels on peut citer Alain Roussillon, La Pensée islamique contemporaine, acteurs et enjeux (Paris, Tétraèdre, coll. "L'islam en débats ", 2005) ; Rachid Benzine, Les Nouveaux penseurs de l'islam (Albin Michel, coll. "Spiritualités ", 2004) ; Franck Frégosi (dir.), Lectures contemporaines du droit islamique. Europe et Monde arabe (Presses universitaires de Strasbourg, 2004); Ibrahim Abu-Rabi', Contemporary Arab Thought: Studies in Post-1967 Arab Intellectual History (Pluto Press, 2004) ; Ghassan Finianos, Islamistes, apologistes et libres penseurs (Presses universitaires de Bordeaux, 2002) ; Farish A. Noor, New Voices of Islam (Leiden, ISIM ed, 2002), John O. Voll et John L. Esposito, Makers of Contemporary Islam (New York, Oxford University Press, 2001) ou encore Charles Kurzman, Liberal Islam: A Source-Book (Oxford University Press, 1998).

3 Comme toute publication qui est le fruit d'une journée d'étude (en novembre 2010), l'ensemble est assez hétérogène. Les articles des différents contributeurs ont néanmoins pu être rassemblés sous trois grandes thématiques: "La figure du Prophète ", "La vision sociale, politique et économique inspirée par la référence religieuse musulmane » et « La figure de la femme ».

4 Après une courte préface ainsi que deux pages de notices biographiques utiles consacrées aux huit personnalités musulmanes dont il est question ici, le recueil s'ouvre sur un article de Felice Dassetto intitulé "Réflexions autour de la pensée musulmane contemporaine » (p. 11-28). Il rappelle brièvement les rôles des pionniers Jamal ad Dîn al-Afghani (1849-1897), Mohammed Abduh (1849-1905) et Rashid Ridâ (1865-1935). Dassetto note ensuite l'important tournant historique qu'a pris en marche la pensée politique islamique: «[...] à partir des années 1920, dans le climat des idéologies totalisantes, fascistes et communistes entre autres [que] des jeunes leaders et intellectuels musulmans sont séduits par cet instrument puissant que représente l'État moderne. Jamais l'islam n'avait eu à sa disposition un instrument aussi puissant 
pour traduire le religieux en réalité sociale"(p.16). Après un court laïus sur les méthodes d'élaboration de la pensée musulmane, il indique Mohammed Charfi et Tariq Ramadan comme étant à la pointe avancée d'une "démarche adaptative" dans la lignée des Frères Musulmans réformistes (p. 25).

Il voit ensuite un groupe constitué par «des démarches issues du mysticisme musulman » (p. 25) comme Faosi Skali (membre de la zawiya Boutchichyia, proche de la monarchie marocaine), le cheikh Khalèd Bentounès (chef spirituel de la zawiya Alwiya) ou Martin Lings (universitaire et soufi, disciple de cette dernière zawiya). Dassetto ajoute Abdennour Bidar à cet ensemble qu'il nomme "fondationisme symbolique ", mais on doute que le prêcheur du "Self Islam " partage suffisamment de points communs avec cette liste de penseurs musulmans contemporains pour en faire partie. Il consacre ensuite un court paragraphe indépendant à l'imam de Bordeaux, Tareq Oubrou, qu'il situe "à la frontière entre le mysticisme et une approche doctrinale " (p. 26), une formule lapidaire mais assez juste.

6 Dassetto entrevoit enfin une quatrième démarche, de type "déconstructrice » (p. 27) ; celle d'individus formés aux sciences humaines et sociales, et qui «investissent les textes fondateurs (Coran et Hadiths) ou de l'histoire fondatrice pour en opérer une révision» (ibid.). Abdelmajid Charfi, Mohammed Arkoun ou encore Daryush Shayegan (disciple d'Henri Corbin) font partie de cette catégorie, ou encore quelques figures du " féminisme musulman » comme Fatima Mernissi, Hidayet Tuksal, Shahla Sherkat ou encore Amina Wadud. L'auteur de cette intéressante contribution liminaire commet par ailleurs une confusion en attribuant le Manifeste pour un islam des Lumières à Rachid Benzine, alors qu'il est l'« œuvre » de Malek Chébel.

7 Bien sûr, cette typologie que propose Felice Dassetto n'a pas pour objectif d'être exhaustive, mais on aurait pu s'attendre à y retrouver les Tunisiens Hicham Djaït, Mohammed Talbi et Yadh Ben Achour, ou, à côté de Mohammed Arkoun (qui est cité), deux autres grands herméneutes de l'islam décédés la même année que lui (2010): l'Égyptien Nasr Hamid Abu Zayd et le marocain Mohammed Abed Al Jabri, qui est peut être allé plus loin qu'Arkoun dans l'historicisation et la tentative de refondation de la pensée musulmane.

L'ouvrage enchaîne ensuite sur sept contributions, puis se clôture sur une conclusion de dix pages de Felice Dassetto. Trois de ces sept contributions (soit près de la moitié) portent sur la figure de Tariq Ramadan. Nous commencerons donc par celles-ci. Khadija Haourigui examine la manière dont l'intellectuel suisse d'origine égyptienne se réfère à la figure du Prophète de l'islam (p. 45-56) dans la biographie qu'il lui a consacrée en 2006, quand Fernand Daniel Dustin s'intéresse au « statut de l'obligation islamique pour les musulmans en Europe» (p. 83) selon Tariq Ramadan (p. 83-100), et Philippe de Briey, enfin, s'appuie sur deux publications récentes de Ramadan pour dégager sa philosophie du pluralisme (p.101-118). Khadija Haourigui observe deux articulations principales dans l'approche de la trajectoire du Prophète par Ramadan: la vie du Prophète comme voie initiatique sur les "questions existentielles, premières et éternelles»(p.48), par ailleurs, "l'attention portée au contexte social, culturel, géographique et politique » de l'époque du Prophète. On perçoit ainsi chez Ramadan des rudiments de contextualisation de cette époque, donc, en principe, de relativisation de l'aspect normatif que celle-ci pourrait constituer pour les musulmans d'aujourd'hui... Même s'il n'y a dans la démarche de Ramadan rien de bien original, elle permet à tout le moins de montrer qu'il ne postule pas un mimétisme aveugle et une 
reproduction des conflits de l'époque bouillonnante de la révélation de l'islam. L'auteur de l'article indique ensuite comment le prophète constitue pour l'intellectuel suisse une figure archétypale de valeurs telles que la générosité et l'équité, l'éthique, la spiritualité, l'éducation, la patience et la résistance, l'amour et la bonté et, de manière moins attendue, la fragilité (p. 49-54).

Fernand Daniel Dustin se focalise, quant à lui, sur le statut des musulmans vivant « en Occident " selon Tariq Ramadan, ainsi que sur sa conception de la si controversée notion de sharîa. L'auteur de cet article note que le premier ouvrage de Ramadan, publié il y a plus de quinze ans, pointe déjà les questions de la présence musulmane en Europe. S'en est suivi une série de quatre ouvrages (p.83) abordant sous différents angles cette thématique. Dustin commence par remarquer que la position de Ramadan telle qu'il l'expose est "paradoxale», puisqu'il plaide pour «la possibilité d'une expression publique du religieux» (p. 84), tout en prônant une certaine adaptation au contexte européen. L'auteur liste d'abord les points positifs - voir les avantages (comme le système scolaire) - que Tariq Ramadan reconnait être ceux des pays européens dont peuvent tirer parti les musulmans d'Europe (droit de fonder des organisations, droit à « la représentation autonome ", droit de faire appel à la loi sans entraves particulières...). Mais l'auteur relève aussitôt que Ramadan pousse les revendications plus loin que ces seuls droits fondamentaux, mais sans détailler. Il évoque ensuite le diagnostic de l'intellectuel suisse qui voit partout en Europe " malaise » et « crise d'identité multidimensionnelle et profonde ».

10 La deuxième partie de cet article, intitulée "les conditions d'élaboration d'une normativité religieuse " est consacrée aux solutions endogènes, internes à l'islam européen, que propose Ramadan. L'auteur a bien examiné les propositions de Ramadan, qu'il décline des pages 91 à 100. Nous avons ici la confirmation de ce que les solutions de Tariq Ramadan ressortissent peut-être plus à la philosophie politique, mâtinée parfois de notions religieuses islamiques (shûra, ijtihâd, bay'a...), mais sans toucher ni aux structures profondes ni superficielles de la pensée islamique classique élaborée au fil des siècles, en situation de dominance politique (et autoritaire) et non pas en situation minoritaire (et démocratique), dans un contexte sécularisé. On sent toujours une certaine réticence chez Tariq Ramadan à s'atteler à une critique de certaines interprétations classiques du Coran par les quatre écoles juridiques classiques ou leurs avatars contemporains, ou à l'implication très contemporaine de certains hadiths problématiques...

11 Enfin, Philippe de Briey résume les «idées nouvelles » qu'il trouve dans l'ouvrage de Tariq Ramadan intitulé Réforme radicale (Presses du Châtelet, 2008), où semble apparaître les linéaments d'un universalisme humaniste à base islamique, rejetant le dogmatisme ou prônant une bonne entente entre les religions (dites " du Livre ", à tout le moins). Il répond plus ou moins à l'épineuse question de la difficulté de séparer religion et politique dans la pensée islamique classique (particulièrement chez les Frères Musulmans dont il est l'héritier) par l'usage de la notion d'« éthique religieuse ", dont il explique qu'elle ne saurait être dissociée des champs de «la politique, de l'économie, de la communication, de la citoyenneté, etc. » (p. 109).

Si nous reprenons l'ouvrage collectif dans l'ordre, à la suite du panorama introductif de Dassetto, un article d'Olivier Abdessalam Ralet propose un aperçu de la conception du Prophète de l'islam, Muhammad, et de la Révélation par Martin Lings et Abdelmajid Charfi (p.29-43), agrémenté de considérations philosophiques qui dénotent une 
réflexion plus personnelle, élaborée et très intéressante sur les thèmes abordés (la figure du Prophète, la philosophie du temps, approche historico-critique et approche apologétique, etc.). La comparaison pourrait de prime abord paraître incongrue, mais Olivier Abdessalam Ralet résume le bien-fondé de sa démarche (p.30) en ce que nous avons ici affaire à un "Occidental tourné vers l'Orient" (l'Anglais Lings) et un "Oriental tourné vers l'Occident» (le Tunisien Charfi). Ralet relève entre les deux auteurs des approches assez dissemblables : chez Lings (islamologue converti à un islam de type soufi), le merveilleux et le miraculeux ont leur place, tandis que Charfi (intellectuel tunisien) refuse tout ce qui selon lui relève de la "pensée magique " (p. 35). Avant une partie conclusive relative au rapport à la modernité de chacun des intellectuels musulmans, Ralet s'autorise un jugement plus personnel et subjectif, où il semble remettre en question l'approche de Charfi: "purifier le message de la révélation de ses références au sacré, au mystère et à l'invisible, comme une forme "exclusiviste" de pensée moderne en général, et Charfi en particulier, entendent le faire, n'est-ce pas "jeter le bébé avec l'eau du bain" ? La spiritualité existe à travers ce qui lui donne chair, les frémissements de la ferveur et les frissons de l'amour qui parcourent les croyants, qui ne relèvent pas de la rationalité postcartésienne, où concepts et affects sont séparés. » (p. 41) Cette réserve de Ralet ne porte pas tant sur le plan scientifique que sur l'effet que la pensée historico-critique peut produire sur le plan spirituel. Au final, l'on sent bien que Ralet - lui-même converti à l'islam comme l'indique sa notule biographique - est plus proche de l'«Occidental tourné vers l'Orient » que de l'« Oriental tourné vers l'Occident ».

13 La quatrième contribution représente le point de jonction entre la première partie consacrée à la figure du Prophète vu par des intellectuels musulmans donc, et la seconde, consacrée aux figures féminines de l'islam contemporain. Yasmina Vanalme s'intéresse en effet à la pensée critique de la sociologue marocaine féministe Fatima Mernissi (p. 57-64), notamment à travers son ouvrage Le Harem Politique. Le Prophète et les femmes (Albin Michel, 1987, et non 1983 comme l'indique l'auteur). On y trouve une approche plus ouvertement critique, non pas de la biographie du Prophète en tant que telle (c'est pour le moment une ligne rouge assez difficilement franchissable chez les intellectuels musulmans), mais ses usages - politiques notamment - qui en sont faits. L'auteur de l'article relève également chez Mernissi une critique de l'authentification des Hadiths (P.59-61), trop nombreux à être canonisés sans suffisamment de précautions.

On poursuit sur le thème femmes et/en islam avec l'étude de Latifa El Hamdi, qui s'intéresse cette fois à quatre auteurs de confession musulmane (p. 65-81): deux théologiens (Tahar Mahdi et Hassan Amdouni), ainsi qu'une auteur-conférencière convertie travaillant dans le milieu de l'éducation (Malika Dif) et une biologiste et intellectuelle marocaine se réclamant du féminisme musulman (Asma Lmrabet). Là encore, la multiplicité des approches - à l'intérieur même de la pensée musulmane que donne à voir cet article est très instructif pour qui considèrerait encore l'islam européen comme un tout homogène strictement prosélyte et conquérant.

Suivent les deux articles sur Tariq Ramadan dont nous avons traité plus haut. Le recueil se clôture par un panorama des "discours économiques de l'islam » par ses leaders et militants (p.119-130). Le texte apparaît quelque peu isolé par rapport au reste de l'ouvrage, mais c'est peut-être parce que le développement de la finance islamique, représentant un nouveau marché qui essaie de s'adosser à la légitimité du registre 
religieux, est relativement récent. Il n'est donc pas dénué d'intérêt de voir quelles dynamiques il produit au sein de la communauté musulmane européenne et qui sont les acteurs qui soutiennent le développement de ce nouveau secteur d'activité.

16 Le directeur de cet ouvrage clôture en essayant de prévoir ce que peut annoncer le grand mouvement de libération du champ discursif et politique qui s'est produit au Sud, connu sous le vocable de "Printemps arabe », pointant l'importance de facteurs extrareligieux comme l'économie, ou la géopolitique (relations Nord-Sud notamment).

17 Quoique de prétention plus limitée que les manuels d'études de la pensée islamique contemporaine (cités ci-dessus), comme l'indique Dassetto lui-même (en préface p. 6), le présent recueil participe utilement à compléter un peu plus ce tour d'horizon dans lequel se sont lancés de nombreux chercheurs en sciences sociales, spécialisés dans l'étude du monde musulman contemporain, ou dans celle de la présence musulmane contemporaine en Europe. 